University of Wollongong

Research Online

Australian Institute for Innovative Materials -

Papers

Australian Institute for Innovative Materials

$1-1-2016$

\title{
Manipulation of nanoscale domain switching using an electron beam with omnidirectional electric field distribution
}

\author{
Zi-Bin Chen \\ University of Sydney \\ Xiaolin Wang \\ University of Wollongong, xiaolin@uow.edu.au \\ Simon Peter Ringer \\ University of Sydney, University of Wollongong \\ Xiaozhou Liao \\ University of Sydney, xliao@usyd.edu.au
}

Follow this and additional works at: https://ro.uow.edu.au/aiimpapers

Part of the Engineering Commons, and the Physical Sciences and Mathematics Commons

\footnotetext{
Research Online is the open access institutional repository for the University of Wollongong. For further information
} contact the UOW Library: research-pubs@uow.edu.au 


\title{
Manipulation of nanoscale domain switching using an electron beam with omnidirectional electric field distribution
}

\author{
Abstract \\ Reversible ferroelectric domain (FD) manipulation with a high spatial resolution is critical for memory \\ storage devices based on thin film ferroelectric materials. FD can be manipulated using techniques that \\ apply heat, mechanical stress, or electric bias. However, these techniques have some drawbacks. Here we \\ propose to use an electron beam with an omnidirectional electric field as a tool for erasable stable \\ ferroelectric nanodomain manipulation. Our results suggest that local accumulation of charges \\ contributes to the local electric field that determines domain configurations.

\section{Keywords} \\ omnidirectional, electric, field, manipulation, distribution, nanoscale, domain, switching, electron, beam

\section{Disciplines} \\ Engineering | Physical Sciences and Mathematics

\section{Publication Details} \\ Chen, Z., Wang, X., Ringer, S. P. \& Liao, X. (2016). Manipulation of nanoscale domain switching using an \\ electron beam with omnidirectional electric field distribution. Physical Review Letters, 117 (2), \\ 027601-1-027601-5.
}




\title{
Manipulation of Nanoscale Domain Switching Using an Electron Beam with Omnidirectional Electric Field Distribution
}

\author{
Zibin Chen, ${ }^{1}$ Xiaolin Wang, ${ }^{2}$ Simon P. Ringer, ${ }^{1,3}$ and Xiaozhou Liao ${ }^{1, *}$ \\ ${ }^{1}$ School of Aerospace, Mechanical and Mechatronic Engineering, The University of Sydney, \\ Sydney, New South Wales 2006, Australia \\ ${ }^{2}$ Institute for Superconducting and Electronic Materials, Faculty of Engineering, Australian Institute for Innovative Materials, \\ University of Wollongong, Wollongong, New South Wales 2522, Australia \\ ${ }^{3}$ Australian Institute for Nanoscale Science and Technology, The University of Sydney, Sydney, New South Wales 2006, Australia
}

(Received 6 December 2015; revised manuscript received 12 April 2016; published 8 July 2016)

\begin{abstract}
Reversible ferroelectric domain (FD) manipulation with a high spatial resolution is critical for memory storage devices based on thin film ferroelectric materials. FD can be manipulated using techniques that apply heat, mechanical stress, or electric bias. However, these techniques have some drawbacks. Here we propose to use an electron beam with an omnidirectional electric field as a tool for erasable stable ferroelectric nanodomain manipulation. Our results suggest that local accumulation of charges contributes to the local electric field that determines domain configurations.
\end{abstract}

DOI: 10.1103/PhysRevLett.117.027601

Ferroelectrics, including typical ferroelectrics and improper ferroelectrics, result from the displacement of ions that leads to spontaneous polarization [1] with the internal electric field pointing towards a specific direction in materials. A region with the same polarization direction is called a ferroelectric domain (FD). With their electrically switchable nature of polarization [2,3], ferroelectric materials [4-6] are important for various types of electronic applications [7-9]. The interest in these materials comes from not only their unique intrinsic piezoelectric and pyroelectric properties, but also the ability of precise manipulation and control of their FDs to the micro- [10] or even nanoscale [11]. Although increasing temperature $[12,13]$ and applying strain [14] both introduce additional energy that could lead to atomic redistribution, it is difficult to use the two methods for precise FD manipulation. Applying an external electric field [15-19] is the easiest way to manipulate FDs at the ambient temperature. This can be achieved using scanning probe microscopes, including the atomic force microscope [20], piezoforce microscope [21], and scanning nonlinear dielectric microscope (SNDM) [19], that work in the contact mode. For example, SNDM has been used to manipulate FDs with a spatial resolution of $\sim 10 \mathrm{~nm}$ [22]. However, the contact-mode leads to serious abrasion of the probe tip and the ferroelectric materials [19]. The problem can be overcome by introducing an electric field using a noncontact mode. The scanning electron microscope (SEM), as a noncontact mode microscope, has been used for the manipulation of FDs. In SEM, electrons are trapped on the sample surface, forming a vertical electric field that changes the local polarization. However, this method usually has a poor patterning resolution [23] due to the nonuniform electron charges through the sample thickness. Yet, the use of SEM to manipulate FDs provides a way of using an electron beam $(e$ beam) for domain patterning.

Here we demonstrate experimentally that an $e$ beam from the transmission electron microscope (TEM) can be used to precisely and reversibly control ferroelectric nanodomain (FND) morphology without contacting the material. An $e$ beam has been widely used for imaging and for local materials heating [24]. However, its capability of generating a controllable local omnidirectional electric field has not been explored. Compared with the existing domain manipulation techniques, TEM presents an ultrahigh spatial resolution of smaller than $\sim 0.2 \mathrm{~nm}$ and local nanoscale charges that generate an electric field along all directions, which potentially allows nanoscale domain writing.

The hexagonal yttrium manganite $\left(\mathrm{YMnO}_{3}\right)$ used in this experiment is improper ferroelectric with corner-sharing $\mathrm{MnO}_{5}$ trigonal bipyramids tilted periodically [25]. The displacement of $\mathrm{Y}$ ions along the $c$ axis or the $\langle 0001\rangle$ direction occurs below its Curie temperature of $\sim 1300 \mathrm{~K}$ [26], leading to the loss of crystal centrosymmetry. The periodic tilting of $\mathrm{MnO}_{5}$ bipyramids enlarges the unit cell by 3 times and leads to the so-called trimerization transition [1], forming three types of antiphase domains $\alpha, \beta$, and $\gamma$. By mutually interlocking with positive and negative FDs, six distinctive domains $\alpha+, \gamma-, \beta+, \alpha-, \gamma+$, and $\beta-$ are alternatively allocated and form a vortexlike domain pattern [1]. Coupling between the trimerization and the displacement effect, $\mathrm{YMnO}_{3}$ consists of only $180^{\circ}$ FDs with the opposite electric polarization direction along the $c$ axis, making it an ideal material for this study.

$E$-beam irradiation and TEM observation were carried out using a JEOL $3000 \mathrm{~F}$ microscope operated at $300 \mathrm{kV}$. The dose rate for the $e$ beam was $\sim 0.07 \mathrm{~A} / \mathrm{cm}^{2}$, which is at a low current density level that would not cause detectable 
(a)

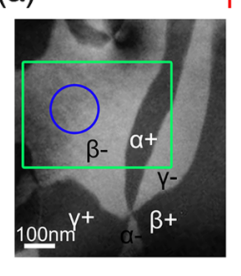

(b)

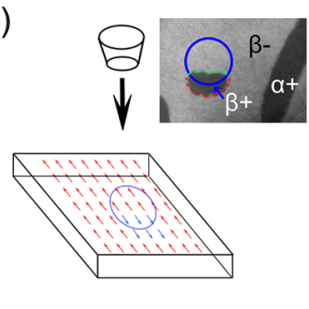

FIG. 1. (a) A dark-field TEM image and its corresponding diffraction pattern. The area that was later subjected to local $e$-beam illumination was extracted from the green box. The blue circle indicates the exact $e$-beam illumination area. The polarization of the white contrast domain $(\beta-)$ is schematically drawn by red arrows. (b) After $e$-beam illumination, a newly formed $\beta+$ domain with dark contrast is seen and its corresponding polarization is shown at the bottom.

sample damage even after long-time (hundreds seconds) $e$-beam illumination [27]. Before the irradiation, the $e$ beam was spread to a large size to prevent major charge accumulation in the observed area. The $e$ beam was then focused on a small area with the required dimension and the illumination time was counted. Once the designed time was reached, the $e$ beam was immediately spread for image acquisition, followed by shutting down the $e$ beam for $10 \mathrm{~min}$ to ensure there is enough time for charge evacuation from the sample. This process was repeated until the maximum designed time was reached.

Figure 1 shows the evolution of a single domain under the $e$ beam in TEM and schematic diagrams that explain the evolution. Figure 1(a) shows a typical dark-field TEM image of a vortexlike domain pattern viewed along a $\langle 1 \overline{1} 00\rangle$ direction and imaged using the $000 \overline{2}$ diffraction spot (circled in the corresponding diffraction pattern). Diffraction contrast images of domains with positive and negative $180^{\circ}$ polarization appear with dark and bright contrast, respectively, under kinematical diffraction conditions [28]. However, the contrast does not differentiate $\alpha, \beta$, and $\gamma$ domains with the same polarization direction. Six distinctive domains $(\alpha+, \beta+, \gamma+$ in dark contrast while $\alpha-, \beta-, \gamma-$ in bright contrast) are seen in Fig. 1(a). The area indicated by a blue circle with a radius of $\sim 60 \mathrm{~nm}$ in the $\beta$ - domain was then exposed to an $e$ beam. Figure 1(b) presents an image of the area marked by the green rectangle in Fig. 1(a) after $e$-beam illumination. A hat-shaped area at the lower part of the blue circle was converted to dark contrast, indicating the redirection of polarization in the area, i.e., the transformation from $\beta-$ to $\beta+$. The redirection occurred because the component of the external electric field along the $z$ axis, $E_{z}$, in the hat-shaped area was equal to or larger than the coercive field $E_{c}$ of the material, and was along the direction opposite to the original polarization.

Figure 2(a) shows another vortexlike domain structure imaged using the $000 \overline{2}$ diffraction spot. After $e$-beam illumination with a radius of $\sim 100 \mathrm{~nm}$ as shown in Fig. 2(b), the $\alpha+$ domain changed its morphology from the original protrusion

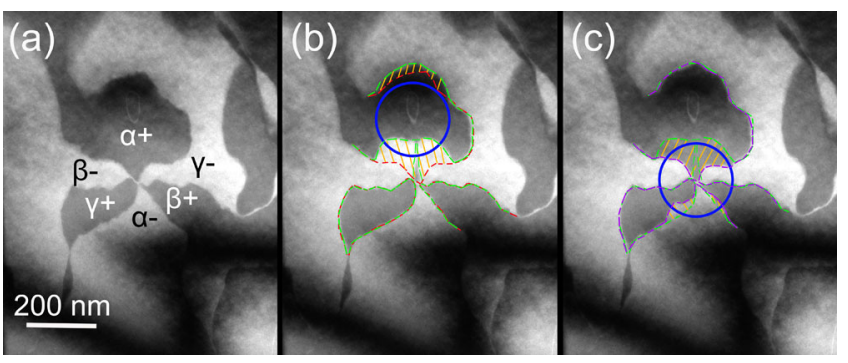

FIG. 2. (a) A dark-field TEM image using the $000 \overline{2}$ diffraction spot. (b) Domain configurations after exposing to the $e$ beam. The blue circle indicates the $e$-beam illumination area. Red and green dashed lines represent the domain boundaries before and after the illumination, respectively. (c) Domain configurations after shifting the $e$ beam to a new position. Green and purple dashed lines represent the domain boundaries before and after the illumination, respectively.

shape (red-dashed lines) to a concave shape (green dash lines) through the shrinkage of the $\alpha+$ domain at the lower part of the blue circle and the slight expansion of the $\alpha+$ domain at the upper part of the blue circle, indicating that the areas at the upper part and the lower part around the $e$-beam illuminated zone evolved in opposite ways. Note that the area with domain switching at the lower part around the $e$-beam illuminated area is larger than that around the upper part of the $e$-beam illuminated area in Fig. 2(b). This is determined by the original domain morphology and the position of the $e$ beam. Interestingly, the expanded $\beta$ - and $\gamma-$ domains did not merge but were separated by a thin antiphase domain wall with dark contrast. An antiphase domain wall provides a defective area avoiding two neighboring domains from merging into one domain and this minimizes the total system energy [29,30]. The moving of antiphase domain walls in this experiment is strong evidence of mutual interlocking [1] of ferroelectric and antiphase domains in $\mathrm{YMnO}_{3}$.

Interestingly, a domain that was exposed to one end of the $e$ beam would return to its original structure if exposed to the opposite end of the $e$ beam, subsequently. This was seen by moving the $e$ beam downward, as shown in Fig. 2(c). The previous expanded $\beta-$ and $\gamma-$ domains (green dashed lines) shown in Fig. 2(b) were then exposed to the upper part of the $e$ beam and this resulted in shrinkage of the $\beta-$ and $\gamma-$ domains and the recovery of the $\alpha+$ domain area (purple dashed lines) to close to its original shape in this area. This result indicates that domain writing using the $e$ beam is erasable. At the same time, the $\alpha$ domain that was exposed to the lower part of the $e$ beam was also expanded, which is similar to the expansion of the $\beta-$ and $\gamma-$ domains shown in Fig. 2(b). Note that the $e$-beam induced domain morphology was stable and remained the same after one month. This stable switching is because of the creation and/or motion of ferroelectric and antiphase domain walls, which provides additional internal energy to resist the relaxation for nanoscale domain recovery.

To explain these phenomena, a theoretical model was built. Figure 3(a) shows a schematic of an $e$ beam 
(a)

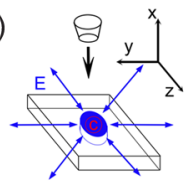

(b)
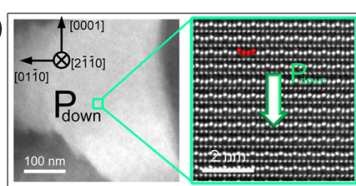

(e)

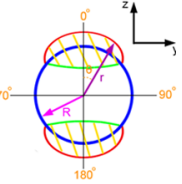

(c)

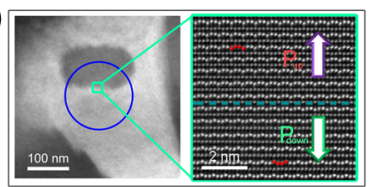

(d)

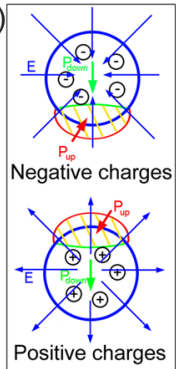

FIG. 3. (a) A schematic showing the omnidirectional electric field $(E)$ induced by $e$-beam illumination in a thin film specimen. The blue circle indicates the $e$ beam illuminated area and the letter "C" represents charges. (b)-(c) TEM (left) and HAADF-STEM (right) images showing polarization before and after $e$-beam irradiation. (d) Schematic drawings of electric fields generated by negative and positive charges. (e) A theoretical model for the electric field magnitude distribution. The areas where domain switching would occur are indicated with yellow shadow bounded by red and green boundary lines in (d) and (e).

illuminating the blue circular area of a thin dielectric film in vacuum. While most of the electrons in the $e$ beam will transmit through the sample, a small amount of charges will be trapped in the thin film through mechanisms that will be discussed below, and the amount of the charges will increase with time before an equilibrium is reached [31]. Both the transmitting electrons and trapped charges contribute to a locally generated omnidirectional electric field.

In the following discussion, a situation is considered in which a ferroelectric material consists of only $180^{\circ} \mathrm{FDs}$ and the polarization directions are defined as parallel to the $\pm \mathrm{z}$ axis. Under these circumstances, a domain structure will be redirected only if the component of the external electric field along the $z$ axis [see Fig. 3(a)] is larger than the coercive field of the material and along the opposite direction of the original polarization [1]. The external electric field components along all other directions perpendicular to the $z$ axis have no effect on redirecting the polarization direction. Therefore, only $E_{z}$ is considered below.

Theoretically, the $z$-axis component of the electric field $\left(E_{z}\right)$ at any position $(r, \theta)$ can be expressed using the following equations [see the Supplemental Material (SI) S1 and S2 [32] for detailed derivation of the equations):

$$
E(r, \theta)_{z}=E_{z_{1}}+E_{z_{2}},
$$

where $E_{z_{1}}$ and $E_{z_{2}}$ are the electric field induced by the continuously passing electrons and trapped charges, respectively.

Theoretical calculation (see SI S2.1 [32]) on the magnitude of the time-independent electric field from continuously passing electrons show that $E_{z_{1}}=2.43 \times$ $10^{-7} \mathrm{kV} / \mathrm{cm}$ for $r=100 \mathrm{~nm}$, which is 8 orders smaller than the coercive field of $40 \mathrm{kV} / \mathrm{cm}$ for $\mathrm{YMnO}_{3}$ [1] and, therefore, is negligible. The electric field contributed by the

trapped charges is therefore the major factor for the domain reorientation.

When an $e$ beam interacts with oxide thin film specimens, there may be a few interaction effects including electron accumulation in the illuminated area [31,36,37], and secondary electron emission [38], and introduction of oxygen vacancies [39]. While electron accumulation leads to negative charges in the $e$-beam illuminated area, the latter two cases introduce positive charges. Therefore, it is important to identify the sign of total trapped charges in the $e$-beam illuminated area. Figure 3(b) shows a TEM image of a domain with bright contrast taken from a $\langle 2 \overline{1} \overline{1} 0\rangle$ zone axis. A high-angle annular dark-field scanning transmission electron microscopy (HAADF-STEM) image taken from the green-square area indicates that the polarization in the domain is down poled $\left(P_{\text {down }}\right)$. Four red dots are marked in Fig. 3(b), which indicate the positions of yttrium ions and these positions are used to identify the polarization direction $[17,40]$. Figure 3(c) shows the result after $e$-beam irradiation at the blue-circled area. Domain switching occurred in the upper part of the irradiation area. The HAADF-STEM image in Fig. 3(c) taken at the domain boundary confirms that the polarization in the dark contrast area is up poled $\left(P_{\text {up }}\right)$, while the polarization in the bright contrast area remains the same.

Effects of electric fields generated by negative and positive charges on domain switching are illustrated in Fig. 3(d). Negative charges result in the electric field directions pointing towards the center of the irradiation area. In this case, the polarization in the lower part of the irradiation area will be reversed. Positive charges lead to the electric field directions pointing outwards, causing domain switching in the upper part of the irradiation area. The experimental results fit the latter case, indicating that the total trapped charges are positive due to the secondary electron emissions and oxygen vacancies introduced by the $e$ beam [41].

Because assuming uniform charge distribution in the $e$-beam illuminated area leads to infinite electric field at the edge of the $e$-beam illuminated area due to the sudden drop of the electrostatic potential at the edge, charges should be distributed in a nonuniform way (but still with the axial symmetry) in the $e$-beam illuminated area. However, the assumption of uniform distribution of charges within the $e$-beam illuminated area can be used to estimate the amount of charges required for domain switching. The calculated value of charge density needed for domain switching is $\sim 1.4 \times 10^{-3} q_{e} / \mathrm{nm}^{2}$ (see SI S2.2 in the Supplemental Material [32]). Gatel et al. reported that $e$-beam illumination on insulated oxide $\mathrm{MgO}$ nanocubes leads to a positive charge density of $8.6 \times 10^{-3} q_{e} / \mathrm{nm}^{2}$ [38], which is 5 times higher than the value of $1.4 \times 10^{-3} q_{e} / \mathrm{nm}^{2}$. The total positive charge density in $\mathrm{YMnO}_{3}$ would be similar to that in $\mathrm{MgO}$ because it led to domain switching. The experimental results that provide the exact information on the switched domain area can be used for data fitting to get the 
value of $E_{z_{2}}$ in Eq. (1) as a function of position and $e$-beam illumination time (see SI S2.3.1 [32]).

Based on the experimental results, the two areas in which $E_{z_{2}} \geq E_{c}$, where $E_{c}$ is the value of the coercive field, in a ferroelectric material is schematically drawn and covered with yellow shadow in Fig. 3(e). $E_{z_{2}}$ in the top and bottom yellow-shadowed areas points to the opposite directions and therefore has opposite effects in redirecting the polarization direction. These are the two areas where the local polarization can be redirected if the local polarization direction is antiparallel to the $E_{z_{2}}$, which explain the phenomena shown in Figs. 1 and 2.

As the value of the charges, which determines the size of the areas with $E_{z_{2}} \geq E_{c}$, is a function of time, the size of a manipulated domain area can be controlled by the $e$-beam illumination duration. A series of focused $e$-beam illumination experiments with the illumination duration set at $10,30,50,100,200$, and $600 \mathrm{~s}$ was carried out [Figs. 4(a)-4(g)]. Figure 4(a) shows the initial morphology of a domain $(\alpha+)$ structure before focused $e$-beam illumination. After focused $e$-beam illumination for $10 \mathrm{~s}$, the morphology remained unchanged [Fig. 4(b)], which indicated the maximum value of $E_{z_{2}}$ at $10 \mathrm{~s}$ is smaller than the coercive field of $40 \mathrm{kV} / \mathrm{cm}$. At $30 \mathrm{~s}$ [Fig. 4(c)], a nanoscale $\alpha$ domain nucleated at the upper part of the $e$-beam illuminated area, as bounded by the hatlike red-dashed lines, indicating that the $E_{z_{2}}$ in the $\alpha$ area is larger than the coercive field of $40 \mathrm{kV} / \mathrm{cm}$. Further increasing the $e$-beam illumination time led to further expansion of the $\alpha$ domain but the expansion rate diminished gradually, as confirmed by the TEM images at 50, 100, 200, and $600 \mathrm{~s}$ in Figs. 4(d) to $4(\mathrm{~g})$. Figure $4(\mathrm{~h})$ illustrates the experimental $E_{z_{2}}-r$ curves (dots) for $e$-beam illumination durations of 30, 50 , 100, 200, and $600 \mathrm{~s}$. Their shapes matched well with the calculated $E_{z_{2}}-r$ curves (solid lines) using equations (s16) when the illumination volume is kept constant, although there were small deviations between different experiments.
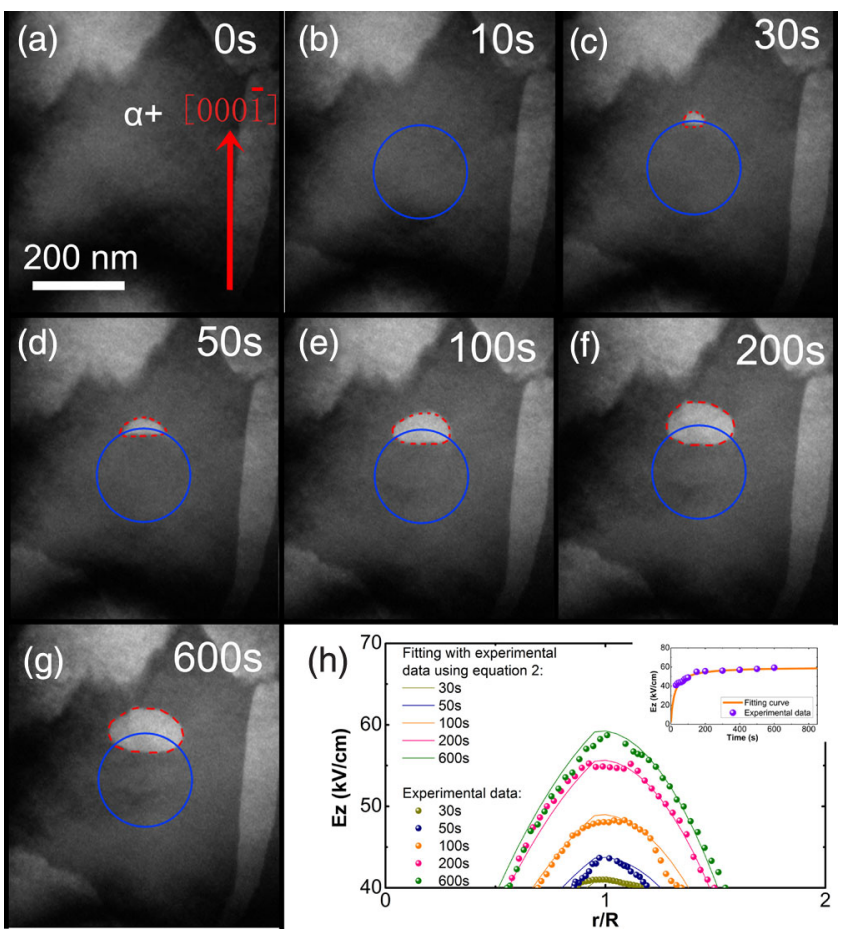

FIG. 4. (a)-(g) A series of dark-field images showing the domain evolution under $e$-beam illumination at $t=0,10,30$, 50, 100, 200, and $600 \mathrm{~s}$, respectively. Blue circles represent the illumination area. The red-dashed curve includes the experimental converted $\alpha$-domain area. (h) Experimental $E_{z}$ versus $r$ plots at different illumination times and the corresponding fitting curves using Eq. (2). The inset shows the fitting curve to the experimental $E_{z}$ as a function of time.

After achieving the $E_{z_{2}}-r$ curves at different e-beam illumination durations, the contribution of trapped charges to $E_{z_{2}}$ as a function of time for any position was achieved as shown in the inset image in Fig. 4(h). Therefore, under the same illumination volume, the $E_{z_{2}}$ at any position $(r, \theta)$ can be expressed using the following equations:

$$
E_{z_{2}}=\left\{\begin{array}{ll}
\left(\frac{5 \mathrm{t}}{(5 \mathrm{t}+100)}\right)\left(93.5\left(\frac{r}{R}\right)-34.9\left(\frac{r}{R}\right)^{2}\right) \cos \theta ; & 0<r \leq R \\
\left(\frac{5 \mathrm{t}}{(5 \mathrm{t}+100)}\right)\left(-23+155.7\left(\frac{r}{R}\right)-75.5\left(\frac{r}{R}\right)^{2}\right) \cos \theta ; & R<r<\infty
\end{array} .\right.
$$

Using these equations, the calculated maximum electric field generated at $10 \mathrm{~s}$ at Fig. 4(b) was $20 \mathrm{kV} / \mathrm{cm}$, which is smaller than the coercive field. Therefore, no domain reorientation occurred at $t=10 \mathrm{~s}$.

By taking advantage of this controllable, erasable, and highly precise positioning technique, domain patterning with high spatial resolution is achievable. Figure 5(a) demonstrates dark-field TEM images of 4 randomly selected original domain areas. With an $e$ beam having a radius of $100 \mathrm{~nm}$, letters $\mathrm{U}, \mathrm{S}, \mathrm{Y}$, and $\mathrm{D}$, which is the abbreviation of the University of Sydney, were written in the 4 areas, respectively [Fig. 5(b)]. Note that some thin white or black straight lines are found passing through the patterns. These lines are antiphase domain walls. To avoid antiphase domain boundaries affecting the pattern quality, poled $\mathrm{YMnO}_{3}$ samples or other ferroelectric materials with large single domain areas can be used.

In conclusion, we demonstrate erasable stable FND manipulation in thin film ferroelectric materials using an $e$ beam. Experimental results and theoretical analysis indicate that the accumulation of positive charges in the thin film plays the key role in generating an electric field for 


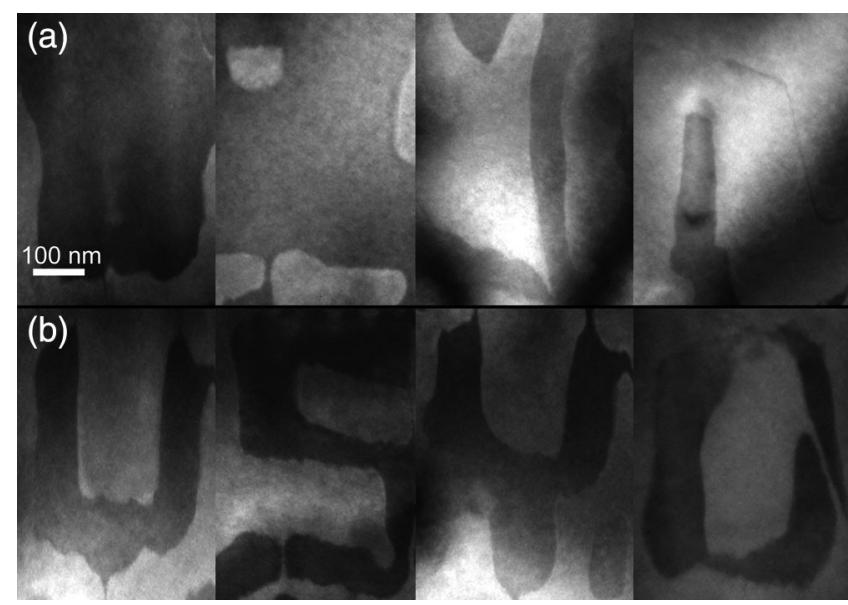

FIG. 5. (a) Initial domain configurations in four areas before $e$-beam illumination. (b) Letters $\mathrm{U}, \mathrm{S}, \mathrm{Y}$, and $\mathrm{D}$ were written on the areas in (a) via $e$-beam illumination.

FND switching. This method requires no prepoling, no electrode, and no physical contact with a tip or any other manipulator and, therefore, would be ideal for manufacturing miniature and integrated electronic devices. Our results also indicate that caution is needed in electron microscopy investigation of FD structures to avoid any artificial effect caused by $e$-beam illumination [40].

The authors are grateful for the scientific and technical support from the Australian Microscopy \& Microanalysis Research Facility node at the University of Sydney and the assistance of Dr. David Mitchell of the Electron Microscopy Centre of the University of Wollongong. We thank the University of Wollongong for providing access to growth facilities used in this work. We thank Mr. Qinyong Lin from the South China University of Technology for the helpful discussion. This research was financially supported by the Australian Research Council.

*To whom all correspondence should be addressed. xiaozhou.liao@sydney.edu.au

[1] T. Choi, Y. Horibe, H. T. Yi, Y. J. Choi, W. D. Wu, and S. W. Cheong, Nat. Mater. 9, 423 (2010).

[2] H. Zhou, Y. M. Pei, F. X. Li, H. S. Luo, and D. N. Fang, Appl. Phys. Lett. 104, 061904 (2014).

[3] W. S. Yun, J. J. Urban, Q. Gu, and H. Park, Nano Lett. 2, 447 (2002).

[4] T. Kimura, T. Goto, H. Shintani, K. Ishizaka, T. Arima, and Y. Tokura, Nature (London) 426, 55 (2003).

[5] I. Grinberg et al., Nature (London) 503, 509 (2013).

[6] W. Eerenstein, N. D. Mathur, and J. F. Scott, Nature (London) 442, 759 (2006).

[7] P. K. Larsen, R. Cuppens, and G. Spierings, Ferroelectrics 128, 265 (1992).

[8] S. T. Han, Y. Zhou, and V. A. L. Roy, Adv. Mater. 25, 5425 (2013).

[9] R. Guo, L. You, Y. Zhou, Z. S. Lim, X. Zou, L. Chen, R. Ramesh, and J. L. Wang, Nat. Commun. 4, 1990 (2013).
[10] L. Mateos, L. E. Bausa, and M. O. Ramirez, Appl. Phys. Lett. 102, 042910 (2013).

[11] X. Z. Chen, Q. Li, X. Chen, X. Guo, H. X. Ge, Y. Liu, and Q. D. Shen, Adv. Funct. Mater. 23, 3124 (2013).

[12] S. C. Chae, N. Lee, Y. Horibe, M. Tanimura, S. Mori, B. Gao, S. Carr, and S. W. Cheong, Phys. Rev. Lett. 108, 167603 (2012).

[13] A. S. Gibbs, K. S. Knight, and P. Lightfoot, Phys. Rev. B 83, 094111 (2011).

[14] P. Gao et al., Nat. Commun. 5, 3801 (2014).

[15] B. Jiang, Y. Bai, W. Y. Chu, Y. J. Su, and L. J. Qiao, Appl. Phys. Lett. 93, 152905 (2008).

[16] Y. Sato, T. Hirayama, and Y. Ikuhara, Phys. Rev. Lett. 107, 187601 (2011).

[17] M. G. Han, Y. M. Zhu, L. J. Wu, T. Aoki, V. Volkov, X. Y. Wang, S. C. Chae, Y. S. Oh, and S. W. Cheong, Adv. Mater. 25, 2415 (2013).

[18] C. T. Nelson et al., Science 334, 968 (2011).

[19] K. Tanaka, Y. Kurihashi, T. Uda, Y. Daimon, N. Odagawa, R. Hirose, Y. Hiranaga, and Y. Cho, Jpn. J. Appl. Phys. 47, 3311 (2008).

[20] H. R. Zeng, K. Shimamura, E. G. Villora, S. Takekawa, and K. Kitamura, J. Mater. Res. 22, 1072 (2007).

[21] S. Matzen, O. Nesterov, G. Rispens, J. A. Heuver, M. Biegalski, H. M. Christen, and B. Noheda, Nat. Commun. 5, 4415 (2014).

[22] Y. S. Cho, J. Mater. Res. 26, 2007 (2011).

[23] J. Li, H. X. Yang, H. F. Tian, C. Ma, S. Zhang, Y. G. Zhao, and J. Q. Li, Appl. Phys. Lett. 100, 152903 (2012).

[24] P. D. Nellist, B. C. McCallum, and J. M. Rodenburg, Nature (London) 374, 630 (1995).

[25] B. B. Van Aken, T. T. M. Palstra, A. Filippetti, and N. A. Spaldin, Nat. Mater. 3, 164 (2004).

[26] S. C. Abrahams, Acta Crystallogr. Sect. B 57, 485 (2001).

[27] R. F. Egerton, P. Li, and M. Malac, Micron 35, 399 (2004).

[28] M. Tanaka, N. Kitamura, and G. Honjo, J. Phys. Soc. Jpn. 17, 1197 (1962).

[29] Y. Kumagai and N. A. Spaldin, Nat. Commun. 4, 1540 (2013).

[30] D. Meier, J. Seidel, A. Cano, K. Delaney, Y. Kumagai, M. Mostovoy, N. A. Spaldin, R. Ramesh, and M. Fiebig, Nat. Mater. 11, 284 (2012).

[31] H. Chen, H. Gong, and C. K. Ong, J. Phys. Condens. Matter 7, 1129 (1995).

[32] See Supplemental Material at http://link.aps.org/ supplemental/10.1103/PhysRevLett.117.027601 for [brief description], which includes Refs. [33-35].

[33] P. M. Whelan and M. J. Hodgson, Essential Principles of Physics (Murray, Cambridge, MA, 1989).

[34] V. E. Cosslett, Nature (London) 309, 186 (1984).

[35] O. Ciftja and I. Hysi, Appl. Math. Lett. 24, 1919 (2011).

[36] I. Mahboob, T. D. Veal, L. F. J. Piper, C. F. McConville, H. Lu, W. J. Schaff, J. Furthmuller, and F. Bechstedt, Phys. Rev. B 69, 201307 (2004).

[37] S. K. Wallace and K. P. McKenna, J. Phys. Chem. C 119, 1913 (2015).

[38] C. Gatel, A. Lubk, G. Pozzi, E. Snoeck, and M. Hytch, Phys. Rev. Lett. 111, 025501 (2013).

[39] C. L. Jia and K. Urban, Science 303, 2001 (2004).

[40] T. Matsumoto et al., Nano Lett. 13, 4594 (2013).

[41] J. Cazaux, J. Electron Spectrosc. Relat. Phenom. 105, 155 (1999). 